\title{
On the $(p, q)$-Humbert Functions from the View Point of the Generating Function Method
}

\author{
Ayman Shehata ${ }^{10}{ }^{1,2}$ \\ ${ }^{1}$ Department of Mathematics, Faculty of Science, Assiut University, Assiut 71516, Egypt \\ ${ }^{2}$ Department of Mathematics, College of Science and Arts, Unaizah, Qassim University, Qassim, Saudi Arabia \\ Correspondence should be addressed to Ayman Shehata; drshehata2006@yahoo.com
}

Received 26 March 2020; Accepted 14 May 2020; Published 25 June 2020

Academic Editor: Serkan Araci

Copyright (c) 2020 Ayman Shehata. This is an open access article distributed under the Creative Commons Attribution License, which permits unrestricted use, distribution, and reproduction in any medium, provided the original work is properly cited.

\begin{abstract}
The main object of the present paper is to construct new $(p, q)$-analogy definitions of various families of $(p, q)$-Humbert functions using the generating function method as a starting point. This study shows a class of several results of $(p, q)$-Humbert functions with the help of the generating functions such as explicit representations and recurrence relations, especially differential recurrence relations, and prove some of their significant properties of these functions.
\end{abstract}

\section{Introduction}

In the last quarter of 20th century, $q$-calculus appeared as a connection between mathematics and physics. We have also a generalization of $q$-calculus with one more parameter, we can say it is a two-parameter quantum calculus. Generally, it is called $(p, q)$-calculus. The theory of $(p, q)$-calculus or post quantum calculus has recently been applied in many areas of mathematics, physics and engineering, such as biology, mechanics, economics, electrochemistry, probability theory, approximation theory, statistics, number theory, quantum theory, theory of relativity, and statistical mechanics, etc. For more details on this topic $(p, q)$-calculus, see, for example, [1-6]. Burban and Klimyk [3], Duran et al. [7-10], Jagannathan [11], Jagannathan and Srinivasa [12], Sahai and Yadav [13] have earlier investigated some properties of the two parameter quantum calculus. Sadjang [14-16] introduced the two $((p, q)$-analogues of the Laplace transform, two $(p, q)$-Taylor formulas for polynomials, $(p, q)$-Appell polynomials and developed some their properties. Mursaleen et al. $[17,18]$ investigated the $(p, q)$-analogues of Bernstein operators and approximation properties of $(p, q)$-Bernstein operators that are a generalization of $q$-Bernstein operators. Khan and Lobiya [19] have nicely discussed a lot of applications in different approximation theory areas, such as per Weirstarass approximation theorems, basic hypergeometric functions, orthogonal polynomials and can be used in differential equations as well as computer-aided geometric designs. Recently, Pasricha and Varma presented and introduced the Humbert function $J_{m, n}(x)$ in $[20,21]$. In [22], Srivastava and Shehata have earlier studied the $q$-Humbert functions. The motivation of these generalizations $q$-Humbert functions is to provide appropriate application areas of mathematical, physical and engineering such as numerical analysis, approximation theory and computer-aided geometric design (see the recent papers $[1,6,19,23]$ and the references therein).

The main purpose of this paper is to obtain explicit formulas for the various families of $(p, q)$-Humbert functions for $0<|q|<|p| \leq 1$ for $p, q$ in $\mathbb{C}$. We mainly use the $(p, q)$-calculus in the theory of special functions. This work is organized as follows. More precisely, we define the numerous (known or new) $(p, q)$-Humbert functions and discuss some significant properties such as explicit representations, recurrence relations and some new generating functions in Section 2. In Section 3, especially recurrence relations and some interesting differential recurrence relations for the $(p, q)$ Humbert functions are discussed. In Section 4, the conclusion and perspectives are given to illustrate the main results.

1.1. Basic Definitions and Miscellaneous Results. To convenience of the reader, we provide a summary of the mathematical notations and some basic definitions of $(p, q)$-calculus 
where $0<|q|<|p| \leq 1$ for $p, q \in \mathbb{C}$, operations and notations we need to be used in this work. We use the following standard notations: $\mathbb{N}=1,2,3, \cdots, \mathbb{N}_{0}=\mathbb{N} \cup\{0\}=\{0,1,2$, $3, \cdots\}$. The symbols $\mathbb{N}$ and $\mathbb{C}$ denote the sets of natural numbers and complex numbers, respectively.

The $q$-number $[\alpha]_{q}$ and $q$-factorial $[n]_{q}$ ! are defined as follows: (see [22])

$$
\begin{gathered}
{[\alpha]_{q}=\frac{1-q^{\alpha}}{1-q}, 0<|q|<1 ; q \in \mathbb{C}-\{1\} ; \alpha \in \mathbb{C}} \\
{[n]_{q} !=\prod_{k=1}^{n}[k]_{q}=[1]_{q}[2]_{q} \cdots[n]_{q}=\prod_{k=1}^{n} \frac{1-q^{k}}{1-q}, q \neq 1 ; n \in \mathbb{N}} \\
q !=1,0<|q|<1 ; q \in \mathbb{C}-\{1\}
\end{gathered}
$$

In [22], the $q$-Humbert functions is defined by

$$
J_{m, n}^{(1)}(x \mid q)=\sum_{k=0}^{\infty} \frac{(-1)^{k}}{[k]_{p, q} ![m+k]_{p, q} ![n+k]_{p, q} !}\left(\frac{x}{3}\right)^{m+n+3 k}
$$

The $(p, q)$-number (bibasic number or twin-basic number) is denoted by $[\alpha]_{p, q}$ and is defined by the following notation [15]

$$
[\alpha]_{p, q}=\frac{p^{\alpha}-q^{\alpha}}{p-q}, 0<|q|<|p| \leq 1 ; p, q, \alpha \in \mathbb{C} .
$$

For $p, q, \alpha \in \mathbb{C}$ and $0<|q|<|p| \leq 1$ for $p, q, \alpha \in \mathbb{C}$, the $(p, q)$-number and $(p, q)$-factorial are given as follows: (see $[11,12,15])$

$$
\begin{aligned}
{[n]_{p, q} } & = \begin{cases}\frac{p^{n}-q^{n}}{p-q}, & n \in \mathbb{N} ; \\
0, & n=0\end{cases} \\
{[n]_{p, q} ! } & =\prod_{k=1}^{n}[k]_{p, q} !=[n]_{p, q}[n-1]_{p, q} \cdots[2]_{p, q}[1]_{p, q}, n \\
& \geq 1 \text { and }[0]_{p, q} !=0 .
\end{aligned}
$$

The $(p, q)$-number $[n]_{p, q}$ is a natural generalization of the $q$-number in (3) such that

$$
\lim _{p \rightarrow 1}[n]_{p, q}=[n]_{q}
$$

The $(p, q)$-number satisfies the following addition properties

$$
\begin{aligned}
{[n]_{p, q} } & =p^{-k}[n+k]_{p, q}-q^{n} p^{-k}[k]_{p, q} \\
& =q^{-k}[n+k]_{p, q}-p^{n} q^{-k}[k]_{p, q}, n, k \in \mathbb{N}
\end{aligned}
$$

The $(p, q)$-factorial is denoted by $[n]_{p, q}$ ! and is defined by (see $[6,11,12])$

$$
[n]_{p, q} != \begin{cases}\prod_{k=1}^{n}[k]_{p, q}=\frac{((p, q) ;(p, q))_{n}}{(p-q)^{n}}, & n \geq 1 \\ 1, & n=0\end{cases}
$$

where

$$
((a, b) ;(p, q))_{n}= \begin{cases}\prod_{r=0}^{n-1}\left(a p^{r}-b q^{r}\right), & n>0 \\ 1, & n=0 \\ \frac{1}{\prod_{r=0}^{-n}\left(a p^{-r}-b q^{-r}\right)}, & n<0 .\end{cases}
$$

As in the $q$-case, there are many definitions of the $(p, q)$ exponential function. The following two $(p, q)$-analogues of exponential function will be frequently used throughout this paper:

The $(p, q)$-exponential function is defined by (see $[12,16])$

$$
e_{p, q}(x)=\sum_{k=0}^{\infty} \frac{p\left(\begin{array}{l}
k \\
2
\end{array}\right) x^{k}}{[k]_{p, q} !}
$$
by

The $(p, q)$-complementary exponential function is defined

$$
E_{p, q}(x)=\sum_{k=0}^{\infty} \frac{q\left(\begin{array}{l}
k \\
2
\end{array}\right) x^{k}}{[k]_{p, q} !}
$$

It is easy to see that (see $[15,16])$

$$
\begin{aligned}
e_{p, q}(x) E_{p, q}(-x) & =1, \\
e_{\bar{p}, \frac{1}{q}}(x) & =E_{p, q}(x), E_{\frac{1}{p}, \frac{1}{q}}(x)=e_{p, q}(x) .
\end{aligned}
$$

Let $f$ be a function defined on a subset of real or complex plane. The $(p, q)$-derivative operator of the function $f$ is defined as follows (see $[15,24,25]$ )

$$
D_{p, q} f(x)=\frac{f(p x)-f(q x)}{(p-q) x}, x \neq 0,
$$

and $\left(D_{p, q} f\right)(0)=f^{\prime}(0)$, provided that $f$ is differentiable at 0 , which satisfies the following relations (see $[14,16]$ )

$$
\begin{aligned}
& D_{p, q} e_{p, q}(\mu x)=a e_{p, q}(\mu p x), \\
& D_{p, q} E_{p, q}(\mu x)=a E_{p, q}(\mu q x), \mu \in \mathbb{C} .
\end{aligned}
$$


The $(p, q)$-derivative operator satisfy the following product rules as follows: (see $[11,12,14,15])$

$$
D_{p, q}\left[f_{1}(x) f_{2}(x)\right]=f_{2}(p x) D_{p, q}\left\{f_{1}(x)\right\}+f_{1}(q x) D_{p, q}\left\{f_{2}(x)\right\}
$$

$$
\begin{aligned}
D_{p, q}\left[f_{1}(x) f_{2}(x) f_{3}(x)\right]= & f_{3}(p x) f_{2}(p x) D_{p, q}\left\{f_{1}(x)\right\} \\
& +f_{3}(p x) f_{1}(q x) D_{p, q}\left\{f_{2}(x)\right\} \\
& +f_{1}(q x) f_{2}(q x) D_{p, q}\left\{f_{3}(x)\right\} .
\end{aligned}
$$

Our purpose is to generalize the class of Bessel functions, by using the same approach exposed above and is to define our main problem on the generalized $(p, q)$-Humbert functions. In particular, we will present some particular cases of functions which are belonging to the family of $(p, q)$-Humbert functions which are introduced as the third $(p, q)$-Humbert functions.

\section{Definitions of $\mathbf{N e w}(p, q)$-Analogue of the $(p$, $q)$-Humbert Functions and Some Basic Properties}

Here we apply the notion of $(p, q)$-analogue of the generating function to obtain explicit formulas for generalized $(p, q)$ Humbert functions and give some interesting significant properties for these functions.

Definition 1. Let us define the product of symmetric $(p, q)$ exponential functions as the generating function of the $(p, q)$-Humbert functions of the first kind as follows:

$$
\begin{aligned}
F_{1}(x ; u, t \mid p, q) & =e_{p, q}\left(\frac{x u}{3}\right) e_{p, q}\left(\frac{x t}{3}\right) e_{p, q}\left(-\frac{x}{3 u t}\right) \\
& =\sum_{m, n=-\infty}^{\infty} J_{m, n}^{(1)}(x \mid p, q) u^{m} t^{n} .
\end{aligned}
$$

Remark 2. Note that in eq. (19), if we put $p=1$, then $(p, q)$ Humbert functions reduces to the q-Humbert functions defined in [22].
Remark 3. When $q \longrightarrow p=1$, the $(\mathrm{p}, \mathrm{q})$-Humbert functions reduce to the classical Humbert functions defined in $[20,21]$.

From (19) and using (11), we have

$$
\begin{aligned}
F_{1}(x ; u, t \mid p, q)= & e_{p, q}\left(\frac{x u}{3}\right) e_{p, q}\left(\frac{x t}{3}\right) e_{p, q}\left(-\frac{x}{3 u t}\right) \\
= & \sum_{r=0}^{\infty} \frac{p^{r(r-1) / 2}}{[r]_{p, q} !}\left(\frac{x u}{3}\right)^{r} \sum_{i=0}^{\infty} \frac{p^{i(i-1) / 2}}{[i]_{p, q} !} \\
& \cdot\left(\frac{x t}{3}\right)^{i} \sum_{k=0}^{\infty} \frac{p^{k(k-1) / 2}}{[k]_{p, q} !}\left(-\frac{x}{3 u t}\right)^{k} \\
= & \sum_{i, r, k=0}^{\infty} \frac{(-1)^{k} p\left(\begin{array}{l}
r \\
2
\end{array}\right)+\left(\begin{array}{l}
i \\
2
\end{array}\right)+\left(\begin{array}{l}
k \\
2
\end{array}\right)}{[k]_{p, q} ![i]_{p, q} ![r]_{p, q} !} \\
& \cdot\left(\frac{x}{3}\right)^{k+i+r} u^{r-k} t^{i-k} .
\end{aligned}
$$

Replace $r$ by $m+k$ and $i$ by $n+k$ to get

$$
\begin{aligned}
& \sum_{m, n=-\infty}^{\infty} \sum_{k=0}^{\infty} \frac{(-1)^{k} p}{[k]_{p, q} ![m+k]_{p, q} ![n+k]_{p, q} !}\left(\frac{c}{m+k}\right)^{m+n+3 k} u^{m} t^{n} \\
& =\sum_{m, n=-\infty}^{\infty} J_{m, n}^{(1)}(x \mid p, q) u^{m} t^{n} .
\end{aligned}
$$

Explicitly, we get the explicit expression of $(p, q)$-Humbert functions $J_{m, n}^{(1)}(x \mid p, q)$ as the following power series

$$
J_{m, n}^{(1)}(x \mid p, q)=\sum_{k=0}^{\infty} \frac{(-1)^{k} p}{[k]_{p, q} ![m+k]_{p, q} ![n+k]_{p, q} !}\left(\frac{c}{m+k}\right)^{m+n+3 k} .
$$

By (9), the series expansions of the $(p, q)$-Humbert functions $J_{m, n}^{(1)}(x \mid p, q)$ are given as

$$
J_{m, n}^{(1)}(x \mid p, q)=\frac{1}{((p, q) ;(p, q))_{n}((p, q) ;(p, q))_{n}} \times \sum_{k=0}^{\infty} \frac{(-1)^{k} p}{((p, q) ;(p, q))_{k}\left(\left(p^{m+1}, q^{m+1}\right) ;(p, q)\right)_{k}\left(\left(p^{n+1}, q^{n+1}\right) ;(p, q)\right)_{k}}\left(\frac{(p-q) x}{3}\right)^{m+n+3 k},
$$


or

$$
J_{m, n}^{(1)}(x \mid p, q)=\frac{1}{\Gamma_{p, q}(m+1) \Gamma_{p, q}(n+1)}\left(\frac{x}{3(p-q)}\right)^{m+n} \times \sum_{k=0}^{\infty} \frac{(-1)^{k} p}{((p, q) ;(p, q))_{k}\left(\left(p^{m+1}, q^{m+1}\right) ;(p, q)\right)_{k}\left(\left(p^{n+1}, q^{n+1}\right) ;(p, q)\right)_{k}}\left(\frac{\left(\begin{array}{c}
m+k \\
2
\end{array}\right)+\left(\begin{array}{c}
n+k \\
2
\end{array}\right)+\left(\begin{array}{c}
k \\
2
\end{array}\right)}{3 k}\right.
$$

equivalently, we have

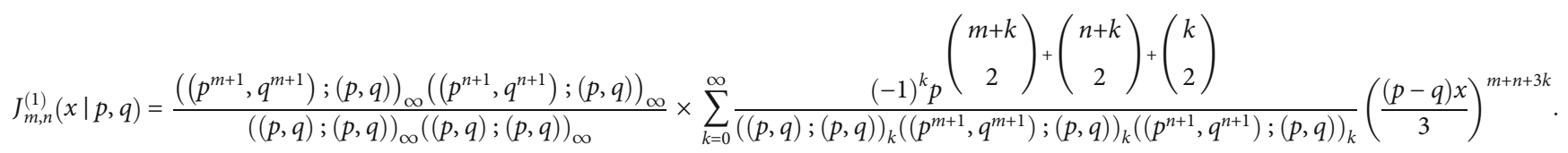

Lemma 4. Let $n$ and $m$ are integers, then the function $J_{m, n}^{(1)}(x \mid p, q)$ satisfies the following relations

$$
\begin{aligned}
& J_{-m, n}^{(1)}(x \mid p, q)=(-1)^{m} J_{m, n+m}^{(1)}(x \mid p, q) \\
& J_{m,-n}^{(1)}(x \mid p, q)=(-1)^{n} J_{m+n, m}^{(1)}(x \mid p, q) .
\end{aligned}
$$

Proof. From the definition of $(p, q)$-Humbert functions $J_{m, n}^{(1)}(x \mid p, q)$, we have

$$
\begin{aligned}
J_{-m, n}^{(1)}(x \mid p, q) & =\sum_{k=0}^{\infty} \frac{(-1)^{k} p}{[k]_{p, q} ![-m+k]_{p, q} ![n+k]_{p, q} !}\left(\begin{array}{c}
-m+k \\
2
\end{array}\right)+\left(\begin{array}{c}
n+k \\
2
\end{array}\right)+\left(\begin{array}{l}
k \\
2
\end{array}\right) \\
& =\sum_{k=m}^{\infty} \frac{(-1)^{k} p}{[k]_{p, q} ![-m+k]_{p, q} ![n+k]_{p, q} !}\left(\begin{array}{c}
-m+k \\
2
\end{array}\right)+\left(\begin{array}{c}
n+k \\
2
\end{array}\right)+\left(\begin{array}{c}
k \\
2
\end{array}\right)
\end{aligned}
$$

Replacing $s=k-m$, we obtain

$$
\begin{aligned}
J_{-m, n}^{(1)}(x \mid p, q)= & \sum_{s=0}^{\infty} \frac{(-1)^{s+m} p}{[s]_{p, q} ![s+m]_{p, q} ![n+m+s]_{p, q} !} \\
& \cdot\left(\frac{x}{3}\right)^{2 m+n+3 s}=(-1)^{m} J_{m, n+m}^{(1)}(x \mid p, q) .
\end{aligned}
$$

Lemma 5. The function $J_{m, n}^{(1)}(x \mid p, q)$ satisfies the following properties

$$
\begin{aligned}
J_{-m,-n}^{(1)}(x \mid p, q) & =(-1)^{m} J_{m, m-n}^{(1)}(x \mid p, q) \\
& =(-1)^{n} J_{n-m, n}^{(1)}(x \mid p, q)
\end{aligned}
$$

where $n$ and $m$ are integers.

Proof. From the definition of $(p, q)$-Humbert functions $J_{m, n}^{(1)}$ $(x \mid p, q)$, we have

$$
\begin{aligned}
J_{-m,-n}^{(1)}(x \mid p, q) & =\sum_{k=0}^{\infty} \frac{(-1)^{k} p}{[k]_{p, q} ![-m+k]_{p, q} ![-n+k]_{p, q} !}\left(\begin{array}{c}
-m+k \\
2
\end{array}\right)+\left(\begin{array}{c}
-n+k \\
2
\end{array}\right)+\left(\begin{array}{l}
k \\
2
\end{array}\right) \\
& =\sum_{k=\max \{n, m\}}^{\infty} \frac{(-1)^{k} p}{[k]_{p, q} ![-m+k]_{p, q} ![-n+k]_{p, q} !}\left(\begin{array}{c}
-m+k \\
2
\end{array}\right)+\left(\begin{array}{c}
-n+k \\
2
\end{array}\right)+\left(\begin{array}{c}
k \\
2
\end{array}\right)
\end{aligned}
$$

Upon setting $s=k-m$ in the Eq. (31), we get

$$
\begin{aligned}
J_{-m,-n}^{(1)}(x \mid p, q) & =\sum_{s=0}^{\infty} \frac{(-1)^{s+m} p}{[s]_{p, q} ![m+s]_{p, q} ![m-n+s]_{p, q} !}\left(\frac{x}{3}\right)^{2 m-n+3 s} \\
& =(-1)^{m} J_{m, m-n}^{(1)}(x \mid p, q) .
\end{aligned}
$$

The equation (27) can be proved in a like manner. 
Upon setting $s=k-n$ in the Eq. (31), we get

$$
\begin{aligned}
J_{-m,-n}^{(1)}(x \mid p, q) & =\sum_{s=0}^{\infty} \frac{(-1)^{s+n} p}{[s]_{p, q} ![n-m+s]_{p, q} ![n+s]_{p, q} !}\left(\begin{array}{c}
n-m+s \\
2
\end{array}\right)+\left(\begin{array}{c}
n+s \\
2
\end{array}\right)+\left(\begin{array}{l}
s \\
2
\end{array}\right) \\
& =(-1)^{n} J_{n-m, n}^{(1)}(x \mid p, q) .
\end{aligned}
$$

Now, we define that the generating function of $(p, q)$ Humbert functions of the second kind.

Definition 6. The generating function $F_{2}(x ; u, t \mid p, q)$ of $(p, q)$-Humbert functions of the second kind is defined by

$$
\begin{aligned}
F_{2}(x ; u, t \mid p, q) & =E_{p, q}\left(\frac{x u}{3}\right) E_{p, q}\left(\frac{x t}{3}\right) E_{p, q}\left(-\frac{q x}{3 u t}\right) \\
& =\sum_{m, n=-\infty}^{\infty} q\left(\begin{array}{l}
n \\
2
\end{array}\right)+\left(\begin{array}{c}
m \\
2
\end{array}\right) J_{m, n}^{(2)}(x \mid p, q) u^{m} t^{n} .
\end{aligned}
$$

$$
\begin{aligned}
= & \sum_{i, r, k=0}^{\infty} \frac{(-1)^{k} q^{r(r-1)+i(i-1)+k(k+1) / 2}}{[k]_{p, q} ![i]_{p, q} ![r]_{p, q} !} \\
& \cdot\left(\frac{x}{3}\right)^{k+i+r} u^{r-k} t^{i-k} .
\end{aligned}
$$

Now, substituting $r$ by $m+k$ and $i$ by $n+k$ in the last equation, we obtain the following equality

$$
\begin{gathered}
\sum_{m, n=-\infty}^{\infty} \sum_{k=0}^{\infty} \frac{(-1)^{k} q^{(m+k)(m+k-1)+(n+k)(n+k-1)+k(k+1) / 2}}{[k]_{p, q} ![m+k]_{p, q} ![n+k]_{p, q} !}\left(\frac{x}{3}\right)^{m+n+3 k} u^{m} t^{n} \\
=\sum_{m, n=-\infty}^{\infty} q^{m(m-1)+n(n-1) / 2} J_{m, n}^{(2)}(x \mid p, q) u^{m} t^{n}
\end{gathered}
$$

From the generating function of the $(p, q)$-Humbert functions $J_{m, n}^{(2)}(x \mid p, q)$, we have

$$
\begin{aligned}
F_{2}(x ; u, t \mid p, q)= & E_{p, q}\left(\frac{x u}{3}\right) E_{p, q}\left(\frac{x t}{3}\right) E_{p, q}\left(-\frac{q x}{3 u t}\right) \\
= & \sum_{r=0}^{\infty} \frac{q^{r(r-1) / 2}}{[r]_{p, q} !}\left(\frac{x u}{3}\right)^{r} \sum_{i=0}^{\infty} \frac{q^{i(i-1) / 2}}{[i]_{p, q} !} \\
& \cdot\left(\frac{x t}{3}\right)^{i} \sum_{k=0}^{\infty} \frac{q^{k(k-1) / 2}}{[k]_{p, q} !}\left(-\frac{q x}{3 u t}\right)^{k}
\end{aligned}
$$

Explicitly, we get the explicit expression of $(p, q)$-Humbert functions $J_{m, n}^{(2)}(x \mid p, q)$ as the following power series

$$
J_{m, n}^{(2)}(x \mid p, q)=\sum_{k=0}^{\infty} \frac{(-1)^{k} q^{1 / 2 k(3 k-1+2(m+n))}}{[k]_{p, q} ![m+k]_{p, q} ![n+k]_{p, q} !}\left(\frac{x}{3}\right)^{m+n+3 k}
$$

equivalently, we have

$$
\begin{aligned}
J_{m, n}^{(2)}(x \mid p, q)= & \frac{1}{((p, q) ;(p, q))_{n}((p, q) ;(p, q))_{n}} \\
& \times \sum_{k=0}^{\infty} \frac{(-1)^{k} q^{1 / 2 k(3 k-1+2(m+n))}}{((p, q) ;(p, q))_{k}\left(\left(p^{m+1}, q^{m+1}\right) ;(p, q)\right)_{k}\left(\left(p^{n+1}, q^{n+1}\right) ;(p, q)\right)_{k}}\left(\frac{(p-q) x}{3}\right)^{m+n+3 k} \\
= & \frac{1}{\Gamma_{p, q}(m+1) \Gamma_{p, q}(n+1)}\left(\frac{x}{3(p-q)}\right)^{m+n} \\
& \times \sum_{k=0}^{\infty} \frac{(-1)^{k} q^{1 / 2 k(3 k-1+2(m+n))}}{((p, q) ;(p, q))_{k}\left(\left(p^{m+1}, q^{m+1}\right) ;(p, q)\right)_{k}\left(\left(p^{n+1}, q^{n+1}\right) ;(p, q)\right)_{k}}\left(\frac{(p-q) x}{3}\right)^{3 k} \\
= & \frac{\left(\left(p^{m+1}, q^{m+1}\right) ;(p, q)\right)_{\infty}\left(\left(p^{n+1}, q^{n+1}\right) ;(p, q)\right)_{\infty}}{((p, q) ;(p, q))_{\infty}((p, q) ;(p, q))_{\infty}} \\
& \times \sum_{k=0}^{\infty} \frac{(-1)^{k} q^{1 / 2 k(3 k-1+2(m+n))}}{((p, q) ;(p, q))_{k}\left(\left(p^{m+1}, q^{m+1}\right) ;(p, q)\right)_{k}\left(\left(p^{n+1}, q^{n+1}\right) ;(p, q)\right)_{k}}\left(\frac{(p-q) x}{3}\right)^{m+n+3 k} .
\end{aligned}
$$


Lemma 7. The connection between generating functions of the $(p, q)$-Humbert functions $J_{m, n}^{(1)}(x \mid p, q)$ and $J_{m, n}^{(2)}(x \mid p, q)$ is given by

$$
J_{m, n}^{(1)}\left(q^{1 / 3} x \mid \frac{1}{p}, \frac{1}{q}\right)=q^{1 / 3(n+m)+}\left(\begin{array}{l}
n \\
2
\end{array}\right)+\left(\begin{array}{c}
m \\
2
\end{array}\right) J_{m, n}^{(2)}(x \mid p, q) .
$$

Proof. If we set that

$$
x=q^{1 / 3} x, t=q^{-1 / 3} t, u=q^{-1 / 3} u
$$

in (19) and using $e_{1 / p, 1 / q}(x)=E_{p, q}(x)$, we get

$$
\begin{gathered}
F_{1}\left(q^{1 / 3} x ; q^{-1 / 3} u, q^{-1 / 3} t \mid \frac{1}{p}, \frac{1}{q}\right)=E_{p, q}\left(\frac{x u}{3}\right) E_{p, q}\left(\frac{x t}{3}\right) E_{p, q}\left(-\frac{q x}{3 u t}\right) \\
\quad=\sum_{m, n=-\infty}^{\infty} J_{m, n}^{(1)}\left(q^{1 / 3} x \mid \frac{1}{p}, \frac{1}{q}\right) q^{-1 / 3(m+n)} u^{m} t^{n}
\end{gathered}
$$

and (34), we obtain (39).

Definition 8. The generating function $F_{3}(x ; u, t \mid p, q)$ of the $(p, q)$-Humbert functions of the third kind $J_{m, n}^{(3)}(x \mid p, q)$ is given by

$$
\begin{aligned}
F_{3}(x ; u, t \mid p, q) & =e_{p, q}\left(\frac{x u}{3}\right) e_{p, q}\left(\frac{x t}{3}\right) E_{p, q}\left(-\frac{q x}{3 u t}\right) \\
& =\sum_{m, n=-\infty}^{\infty} J_{m, n}^{(3)}(x \mid p, q) u^{m} t^{n} .
\end{aligned}
$$

Using (42), (11) and (12), we have

$$
\begin{aligned}
& F_{3}(x ; u, t \mid p, q) \\
& =\sum_{r=0}^{\infty} \frac{p^{r(r-1) / 2}}{[r]_{p, q} !}\left(\frac{x u}{3}\right)^{r} \sum_{i=0}^{\infty} \frac{p^{i(i-1) / 2}}{[i]_{p, q} !}\left(\frac{x t}{3}\right)^{i} \sum_{k=0}^{\infty} \frac{q^{k(k-1) / 2}}{[k]_{p, q} !}\left(-\frac{q x}{3 u t}\right)^{k} \\
& =\sum_{i, r, k=0}^{\infty} \frac{(-1)^{k} p\left(\begin{array}{c}
r \\
2
\end{array}\right)+\left(\begin{array}{l}
i \\
2
\end{array}\right)_{q}\left(\begin{array}{l}
k \\
2
\end{array}\right)}{[k]_{p, q} ![i]_{p, q} ![r]_{p, q} !}\left(\frac{x}{3}\right)^{k+i+r} u^{r-k} t^{i-k} .
\end{aligned}
$$

Substituting $r$ by $m+k$ and $i$ by $n+k$ in the last equation, we obtain the following equality

$$
\begin{aligned}
& \sum_{m, n=-\infty}^{\infty} \sum_{k=0}^{\infty} \frac{(-1)^{k} p}{[k]_{p, q} ![m+k]_{p, q} ![n+k]_{p, q} !}\left(\frac{c}{m+k}\right)^{m+n+3 k} u^{m} t^{n} \\
& =\sum_{m, n=-\infty}^{\infty} J_{m, n}^{(3)}(x \mid p, q) u^{m} t^{n} .
\end{aligned}
$$

Explicitly, we get the explicit expression of $(p, q)$-Humbert functions $J_{m, n}^{(3)}(x \mid p, q)$ of the third kind as the following power series

$$
J_{m, n}^{(3)}(x \mid p, q)=\sum_{k=0}^{\infty} \frac{(-1)^{k} p\left(\begin{array}{c}
m+k \\
2
\end{array}\right)+\left(\begin{array}{c}
n+k \\
2
\end{array}\right){ }_{q}\left(\begin{array}{c}
k+1 \\
2
\end{array}\right)}{[k]_{p, q} ![m+k]_{p, q} ![n+k]_{p, q} !}\left(\frac{x}{3}\right)^{m+n+3 k},
$$

or, equivalently, we get

$$
\begin{aligned}
& J_{m, n}^{(3)}(x \mid p, q)=\frac{1}{((p, q) ;(p, q))_{n}((p, q) ;(p, q))_{n}} \\
& \times \sum_{k=0}^{\infty} \frac{(-1)^{k} p\left(\begin{array}{c}
m+k \\
2
\end{array}\right)+\left(\begin{array}{c}
n+k \\
2
\end{array}\right)\left(\begin{array}{c}
k+1 \\
2
\end{array}\right)}{(p, q) ;(p, q))_{k}\left(\left(p^{m+1}, q^{m+1}\right) ;(p, q)\right)_{k}\left(\left(p^{n+1}, q^{n+1}\right) ;(p, q)\right)_{k}}\left(\frac{(p-q) x}{3}\right)^{m+n+3 k} \\
& =\frac{1}{\Gamma_{p, q}(m+1) \Gamma_{p, q}(n+1)}\left(\frac{x}{3(p-q)}\right)^{m+n} \\
& \times \sum_{k=0}^{\infty} \frac{(-1)^{k} p\left(\begin{array}{c}
m+k \\
2
\end{array}\right)+\left(\begin{array}{c}
n+k \\
2
\end{array}\right)_{q}\left(\begin{array}{c}
k+1 \\
2
\end{array}\right)}{((p, q) ;(p, q))_{k}\left(\left(p^{m+1}, q^{m+1}\right) ;(p, q)\right)_{k}\left(\left(p^{n+1}, q^{n+1}\right) ;(p, q)\right)_{k}}\left(\frac{(p-q) x}{3}\right)^{3 k} \\
& =\frac{\left(\left(p^{m+1}, q^{m+1}\right) ;(p, q)\right)_{\infty}\left(\left(p^{n+1}, q^{n+1}\right) ;(p, q)\right)_{\infty}}{((p, q) ;(p, q))_{\infty}((p, q) ;(p, q))_{\infty}} \\
& \times \sum_{k=0}^{\infty} \frac{(-1)^{k} p\left(\begin{array}{c}
m+k \\
2
\end{array}\right)+\left(\begin{array}{c}
n+k \\
2
\end{array}\right)_{q}\left(\begin{array}{c}
k+1 \\
2
\end{array}\right)}{((p, q) ;(p, q))_{k}\left(\left(p^{m+1}, q^{m+1}\right) ;(p, q)\right)_{k}\left(\left(p^{n+1}, q^{n+1}\right) ;(p, q)\right)_{k}}\left(\frac{(p-q) x}{3}\right)^{m+n+3 k} .
\end{aligned}
$$


Definition 9. A fourth generating function $F_{4}(x ; u, t \mid p, q)$ of the $(p, q)$-Humbert functions $J_{m, n}^{(4)}(x \mid p, q)$ of the fourth kind is defined by

$$
\begin{aligned}
F_{4}(x ; u, t \mid p, q) & =E_{p, q}\left(\frac{q x u}{3}\right) E_{p, q}\left(\frac{q x t}{3}\right) e_{p, q}\left(-\frac{x}{3 u t}\right) \\
& =\sum_{m, n=-\infty}^{\infty} J_{m, n}^{(4)}(x \mid p, q) u^{m} t^{n}
\end{aligned}
$$

Using (47), (11) and (12), we have

$$
\begin{aligned}
F_{4}(x ; u, t \mid p, q)= & \sum_{r=0}^{\infty} \frac{q^{r(r-1) / 2}}{[r]_{p, q} !}\left(\frac{q x u}{3}\right)^{r} \sum_{i=0}^{\infty} \frac{q^{i(i-1) / 2}}{[i]_{p, q} !}\left(\frac{q x t}{3}\right)^{i} \\
& \cdot \sum_{k=0}^{\infty} \frac{p^{k(k-1) / 2}}{[k]_{p, q} !}\left(-\frac{x}{3 u t}\right)^{k}
\end{aligned}
$$

$$
\begin{aligned}
= & \sum_{i, r, k=0}^{\infty} \frac{(-1)^{k} q}{\left(\begin{array}{l}
r \\
2
\end{array}\right)+\left(\begin{array}{l}
i \\
2
\end{array}\right)}\left(\begin{array}{l}
k \\
2
\end{array}\right) q^{r+i} \\
& \cdot\left(\frac{x}{3}\right)^{k+i+r} ! u^{r-k} t^{i-k} .
\end{aligned}
$$

Replace $r$ by $m+k$ and $i$ by $n+k$ to get

$$
\begin{aligned}
& \sum_{m, n=-\infty}^{\infty} \sum_{k=0}^{\infty} \frac{(-1)^{k} q}{[k]_{p, q} ![m+k]_{p, q} ![n+k]_{p, q} !}\left(\begin{array}{c}
m+k+1 \\
2
\end{array}\right)+\left(\begin{array}{c}
n+k+1 \\
3
\end{array}\right)^{(}\left(\begin{array}{l}
k \\
2
\end{array}\right) \\
& =\sum_{m, n=-\infty}^{\infty} J_{m, n}^{(4)}(x \mid p, q) u^{m} t^{n} .
\end{aligned}
$$

Explicitly, we obtain the explicit expressions of $(p, q)$ -Humbert functions $J_{m, n}^{(4)}(x \mid p, q)$ as

$$
\begin{aligned}
& J_{m, n}^{(4)}(x \mid p, q)=\sum_{k=0}^{\infty} \frac{(-1)^{k} p}{[k]_{p, q} ![m+k]_{p, q} ![n+k]_{p, q} !}\left(\frac{l}{3}\right)^{m+n+3 k} \\
& =\frac{1}{((p, q) ;(p, q))_{n}((p, q) ;(p, q))_{n}} \\
& \times \sum_{k=0}^{\infty} \frac{(-1)^{k} p}{((p, q) ;(p, q))_{k}\left(\left(p^{m+1}, q^{m+1}\right) ;(p, q)\right)_{k}\left(\left(p^{n+1}, q^{n+1}\right) ;(p, q)\right)_{k}}\left(\frac{(p-q) x}{3}\right)^{m+n+3 k} \\
& =\frac{1}{\Gamma_{p, q}(m+1) \Gamma_{p, q}(n+1)}\left(\frac{x}{3(p-q)}\right)^{m+n}
\end{aligned}
$$

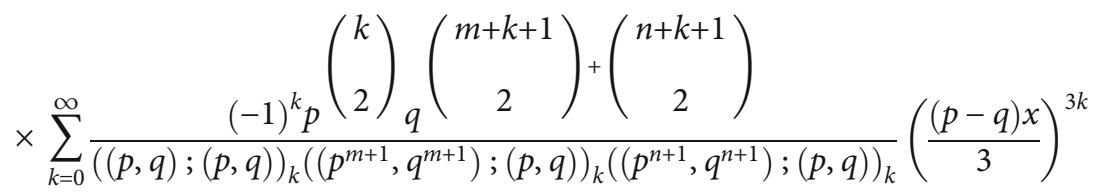

$$
\begin{aligned}
& =\frac{\left(\left(p^{m+1}, q^{m+1}\right) ;(p, q)\right)_{\infty}\left(\left(p^{n+1}, q^{n+1}\right) ;(p, q)\right)_{\infty}}{((p, q) ;(p, q))_{\infty}((p, q) ;(p, q))_{\infty}}
\end{aligned}
$$

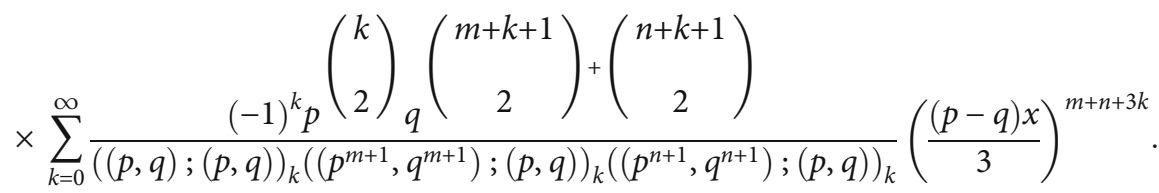


Definition 10. The generating function $F_{5}(x ; u, t \mid p, q)$ of the $(p, q)$-Humbert functions $J_{m, n}^{(5)}(x \mid p, q)$ of the fifth kind is defined as

$$
\begin{aligned}
F_{5}(x ; u, t \mid p, q) & =e_{p, q}\left(\frac{x u}{3}\right) E_{p, q}\left(\frac{q x t}{3}\right) E_{p, q}\left(-\frac{q x}{3 u t}\right) \\
& =\sum_{m, n=-\infty}^{\infty} q^{\frac{1}{2} n(n-1)} J_{m, n}^{(5)}(x \mid p, q) u^{m} t^{n}
\end{aligned}
$$

Using (11) (12) and (51), we have

$$
\begin{gathered}
F_{5}(x ; u, t \mid p, q) \\
=\sum_{r=0}^{\infty} \frac{p^{r(r-1) / 2}}{[r]_{p, q} !}\left(\frac{x u}{3}\right)^{r} \sum_{i=0}^{\infty} \frac{q^{i(i-1) / 2}}{[i]_{p, q} !} \\
\cdot\left(\frac{q x t}{3}\right)^{i} \sum_{k=0}^{\infty} \frac{q^{k(k-1) / 2}}{[k]_{p, q} !}\left(-\frac{q x}{3 u t}\right)^{k}
\end{gathered}
$$

$$
=\sum_{i, r, k=0}^{\infty} \frac{(-1)^{k} p\left(\begin{array}{l}
r \\
2
\end{array}\right)\left(\begin{array}{l}
i \\
2
\end{array}\right)+\left(\begin{array}{l}
k \\
2
\end{array}\right) q^{i+k}}{[k]_{p, q} ![i]_{p, q} ![r]_{p, q} !}\left(\frac{x}{3}\right)^{k+i+r} u^{r-k} t^{i-k} .
$$

Upon setting $r=m+k$ and $i-n+k$ in the above equation, we get

$$
\begin{aligned}
& \sum_{m, n=-\infty}^{\infty} \sum_{k=0}^{\infty} \frac{(-1)^{k} p}{[k]_{p, q} ![m+k]_{p, q} ![n+k]_{p, q} !}\left(\begin{array}{c}
m+k \\
2
\end{array}\right)\left(\begin{array}{c}
n+k+1 \\
3
\end{array}\right)^{m+n+3 k} u^{m} t^{n} \\
& =\sum_{m, n=-\infty}^{\infty} q\left(\begin{array}{c}
k+1 \\
2
\end{array}\right)_{J_{m, n}^{(5)}(x \mid p, q) u^{m} t^{n} .}
\end{aligned}
$$

Explicitly, we obtain the explicit representations of $(p, q)$ -Humbert functions $J_{m, n}^{(5)}(x \mid p, q)$ of the fifth kind as the following power series

$$
\begin{aligned}
& J_{m, n}^{(5)}(x \mid p, q)=\sum_{k=0}^{\infty} \frac{(-1)^{k} p\left(\begin{array}{c}
m+k \\
2
\end{array}\right)}{[k]_{p, q} ![m+k]_{p, q} ![n+k]_{p, q} !}\left(\begin{array}{c}
n+k+1 \\
2
\end{array}\right)+\left(\begin{array}{c}
k+1 \\
2
\end{array}\right)-\left(\begin{array}{l}
n \\
2
\end{array}\right)^{m+n+3 k} \\
& =\frac{1}{((p, q) ;(p, q))_{n}((p, q) ;(p, q))_{n}} \\
& \times \sum_{k=0}^{\infty} \frac{(-1)^{k} p}{((p, q) ;(p, q))_{k}\left(\left(p^{m+1}, q^{m+1}\right) ;(p, q)\right)_{k}\left(\left(p^{n+1}, q^{n+1}\right) ;(p, q)\right)_{k}}\left(\frac{(p-q) x}{3}\right)^{m+n+3 k} \\
& =\frac{1}{\Gamma_{p, q}(m+1) \Gamma_{p, q}(n+1)}\left(\frac{x}{3(p-q)}\right)^{m+n} \\
& \times \sum_{k=0}^{\infty} \frac{(-1)^{k} p}{((p, q) ;(p, q))_{k}\left(\left(p^{m+1}, q^{m+1}\right) ;(p, q)\right)_{k}\left(\left(p^{n+1}, q^{n+1}\right) ;(p, q)\right)_{k}}\left(\frac{(p-q) x}{3}\right)^{3 k} \\
& =\frac{\left(\left(p^{m+1}, q^{m+1}\right) ;(p, q)\right)_{\infty}\left(\left(p^{n+1}, q^{n+1}\right) ;(p, q)\right)_{\infty}}{((p, q) ;(p, q))_{\infty}((p, q) ;(p, q))_{\infty}} \\
& \times \sum_{k=0}^{\infty} \frac{(-1)^{k} p}{((p, q) ;(p, q))_{k}\left(\left(p^{m+1}, q^{m+1}\right) ;(p, q)\right)_{k}\left(\left(p^{n+1}, q^{n+1}\right) ;(p, q)\right)_{k}}\left(\frac{(p-q) x}{3}\right)^{m+n+3 k} .
\end{aligned}
$$


Definition 11. The generating function $F_{6}(x ; u, t \mid p, q)$ of the $(p, q)$-Humbert functions $J_{m, n}^{(6)}(x \mid p, q)$ of the sixth kind is defined by

$$
\begin{aligned}
F_{6}(x ; u, t \mid p, q) & =E_{p, q}\left(\frac{q x u}{3}\right) e_{p, q}\left(\frac{x t}{3}\right) E_{p, q}\left(-\frac{q x}{3 u t}\right) \\
& =\sum_{m, n=-\infty}^{\infty} q^{\frac{1}{2} m(m-1)} J_{m, n}^{(6)}(x \mid p, q) u^{m} t^{n}
\end{aligned}
$$

From (55), (11) and (12), we have

$$
\begin{aligned}
F_{6}(x ; u, t \mid p, q)= & \sum_{r=0}^{\infty} \frac{q^{r(r-1) / 2}}{[r]_{p, q} !}\left(\frac{q x u}{3}\right)^{r} \sum_{i=0}^{\infty} \frac{p^{i(i-1) / 2}}{[i]_{p, q} !} \\
& \cdot\left(\frac{x t}{3}\right)^{i} \sum_{k=0}^{\infty} \frac{q^{k(k-1) / 2}}{[k]_{p, q} !}\left(-\frac{q x}{3 u t}\right)^{k}
\end{aligned}
$$

$$
=\sum_{i, r, k=0}^{\infty} \frac{(-1)^{k} p\left(\begin{array}{l}
i \\
2
\end{array}\right)\left(\begin{array}{l}
r \\
2
\end{array}\right)+\left(\begin{array}{l}
k \\
2
\end{array}\right) q^{r+k}}{[k]_{p, q} ![i]_{p, q} ![r]_{p, q} !}\left(\frac{x}{3}\right)^{k+i+r} u^{r-k} t^{i-k} .
$$

Substituting $r$ by $m+k$ and $i$ by $n+k$ in the last equation, we get the following equality

$$
\begin{aligned}
& \sum_{m, n=-\infty}^{\infty} \sum_{k=0}^{\infty} \frac{(-1)^{k} p}{[k]_{p, q} ![m+k]_{p, q} ![n+k]_{p, q} !}\left(\begin{array}{c}
n+k \\
2
\end{array}\right)\left(\begin{array}{c}
m+k+1 \\
2
\end{array}\right)^{m+n+3 k} u^{m} t^{n} \\
& =\sum_{m, n=-\infty}^{\infty} q\left(\begin{array}{c}
k+1 \\
2
\end{array}\right)_{J_{m, n}^{(6)}(x \mid p, q) u^{m} t^{n} .}
\end{aligned}
$$

Explicitly, we obtain the explicit representations of $(p, q)$ Humbert functions $J_{m, n}^{(6)}(x \mid p, q)$ of the sixth kind as the following power series

$$
\begin{aligned}
& J_{m, n}^{(6)}(x \mid p, q)=\sum_{k=0}^{\infty} \frac{(-1)^{k} p\left(\begin{array}{c}
n+k \\
2
\end{array}\right) q_{q}\left(\begin{array}{c}
m+k+1 \\
2
\end{array}\right)+\left(\begin{array}{c}
k+1 \\
2
\end{array}\right)-\left(\begin{array}{c}
m \\
2
\end{array}\right)}{[k]_{p, q} ![m+k]_{p, q} ![n+k]_{p, q} !}\left(\frac{x}{3}\right)^{m+n+3 k} \\
& =\frac{1}{((p, q) ;(p, q))_{n}((p, q) ;(p, q))_{n}} \\
& \times \sum_{k=0}^{\infty} \frac{(-1)^{k} p}{((p, q) ;(p, q))_{k}\left(\left(p^{m+1}, q^{m+1}\right) ;(p, q)\right)_{k}\left(\left(p^{n+1}, q^{n+1}\right) ;(p, q)\right)_{k}}\left(\frac{(p-q) x}{3}\right)^{m+n+3 k} \\
& =\frac{1}{\Gamma_{p, q}(m+1) \Gamma_{p, q}(n+1)}\left(\frac{x}{3(p-q)}\right)^{m+n} \\
& \times \sum_{k=0}^{\infty} \frac{(-1)^{k} p}{((p, q) ;(p, q))_{k}\left(\left(p^{m+1}, q^{m+1}\right) ;(p, q)\right)_{k}\left(\left(p^{n+1}, q^{n+1}\right) ;(p, q)\right)_{k}}\left(\frac{(p-q) x}{3}\right)^{3 k} \\
& =\frac{\left(\left(p^{m+1}, q^{m+1}\right) ;(p, q)\right)_{\infty}\left(\left(p^{n+1}, q^{n+1}\right) ;(p, q)\right)_{\infty}}{((p, q) ;(p, q))_{\infty}((p, q) ;(p, q))_{\infty}} \\
& \times \sum_{k=0}^{\infty} \frac{(-1)^{k} p}{((p, q) ;(p, q))_{k}\left(\left(p^{m+1}, q^{m+1}\right) ;(p, q)\right)_{k}\left(\left(p^{n+1}, q^{n+1}\right) ;(p, q)\right)_{k}}\left(\frac{(p-q) x}{3}\right)^{m+n+3 k} .
\end{aligned}
$$


Definition 12. The generating function $F_{7}(x ; u, t \mid p, q)$ of the $(p, q)$-Humbert functions $J_{m, n}^{(7)}(x \mid p, q)$ of the seventh kind is defined by

$$
\begin{aligned}
F_{7}(x ; u, t \mid p, q) & =e_{p, q}\left(\frac{x u}{3}\right) E_{p, q}\left(\frac{q x t}{3}\right) e_{p, q}\left(-\frac{x}{3 u t}\right) \\
& =\sum_{m, n=-\infty}^{\infty} J_{m, n}^{(7)}(x \mid p, q) u^{m} t^{n} .
\end{aligned}
$$

From (11), (12) and ((59), we have

$$
\begin{aligned}
F_{7}(x ; u, t \mid p, q)= & \sum_{r=0}^{\infty} \frac{p^{r(r-1) / 2}}{[r]_{p, q} !}\left(\frac{x u}{3}\right)^{r} \sum_{i=0}^{\infty} \frac{q^{i(i-1) / 2}}{[i]_{p, q} !} \\
& \cdot\left(\frac{q x t}{3}\right)^{i} \sum_{k=0}^{\infty} \frac{p^{k(k-1) / 2}}{[k]_{p, q} !}\left(-\frac{x}{3 u t}\right)^{k}
\end{aligned}
$$

$$
=\sum_{i, r, k=0}^{\infty} \frac{(-1)^{k} q_{p}^{\left(\begin{array}{l}
i \\
2
\end{array}\right)}\left(\begin{array}{l}
r \\
2
\end{array}\right)+\left(\begin{array}{l}
k \\
2
\end{array}\right)}{[k]_{p, q} ![i]_{p, q} ![r]_{p, q} !}\left(\frac{x}{3}\right)^{k+i+r} u^{r-k} t^{i-k} .
$$

Replacing $r$ by $m+k$ and $i$ by $n+k$ in the above equation, we obtain the following equality

$$
\begin{aligned}
& \sum_{m, n=-\infty}^{\infty} \sum_{k=0}^{\infty} \frac{(-1)^{k} q}{[k]_{p, q} ![m+k]_{p, q} ![n+k]_{p, q} !}\left(\frac{\left(\begin{array}{c}
n+k+1 \\
3
\end{array}\right)^{m+n+3 k} u^{m} t^{n}}{2}\left(\begin{array}{c}
m+k \\
2
\end{array}\right)+\left(\begin{array}{l}
k \\
2
\end{array}\right)\right. \\
& =\sum_{m, n=-\infty}^{\infty} J_{m, n}^{(7)}(x \mid p, q) u^{m} t^{n} .
\end{aligned}
$$

Explicitly, we get the explicit expressions of $(p, q)$-Humbert functions $J_{m, n}^{(7)}(x \mid p, q)$ of the seventh kind as the following power series

$$
\begin{aligned}
& J_{m, n}^{(7)}(x \mid p, q)=\sum_{k=0}^{\infty} \frac{(-1)^{k} q}{[k]_{p, q} ![m+k]_{p, q} ![n+k]_{p, q} !}\left(\frac{c}{n+k+1} 2\right)^{m+n+3 k} \\
& J_{m, n}^{(7)}(x \mid p, q)=\frac{1}{((p, q) ;(p, q))_{n}((p, q) ;(p, q))_{n}}
\end{aligned}
$$

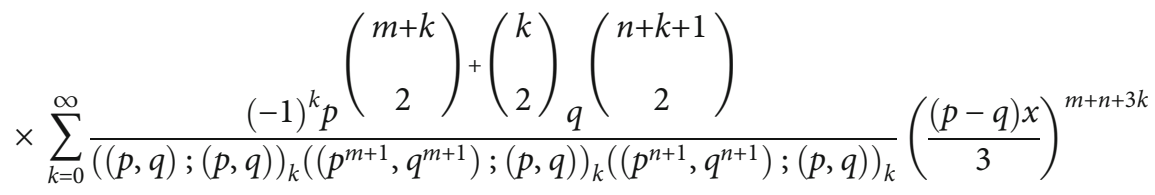

$$
\begin{aligned}
& =\frac{1}{\Gamma_{p, q}(m+1) \Gamma_{p, q}(n+1)}\left(\frac{x}{3(p-q)}\right)^{m+n} \\
& \times \sum_{k=0}^{\infty} \frac{(-1)^{k} p}{((p, q) ;(p, q))_{k}\left(\left(^{m+1}, q^{m+1}\right) ;(p, q)\right)_{k}\left(\left(p^{n+1}, q^{n+1}\right) ;(p, q)\right)_{k}}\left(\frac{(p-q) x}{3}\right)^{3 k} \\
& =\frac{\left(\left(p^{m+1}, q^{m+1}\right) ;(p, q)\right)_{\infty}\left(\left(p^{n+1}, q^{n+1}\right) ;(p, q)\right)_{\infty}}{((p, q) ;(p, q))_{\infty}((p, q) ;(p, q))_{\infty}} \\
& \times \sum_{k=0}^{\infty} \frac{(-1)^{k} p}{((p, q) ;(p, q))_{k}\left(\left(^{m+1}, q^{m+1}\right) ;(p, q)\right)_{k}\left(\left(p^{n+1}, q^{n+1}\right) ;(p, q)\right)_{k}}\left(\frac{(p-q) x}{3}\right)^{m+n+3 k} .
\end{aligned}
$$


Definition 13. The generating function $F_{8}(x ; u, t \mid p, q)$ of the $(p, q)$-Humbert functions $J_{m, n}^{(8)}(x \mid p, q)$ of the eighth kind is defined by

$$
\begin{aligned}
F_{8}(x ; u, t \mid p, q) & =E_{p, q}\left(\frac{q x u}{3}\right) e_{p, q}\left(\frac{x t}{3}\right) e_{p, q}\left(-\frac{x}{3 u t}\right) \\
& =\sum_{m, n=-\infty}^{\infty} J_{m, n}^{(8)}(x \mid p, q) u^{m} t^{n} .
\end{aligned}
$$

From (11), (12) and (63), we have

$$
\begin{aligned}
F_{8}(x ; u, t \mid p, q)= & \sum_{r=0}^{\infty} \frac{q^{r(r-1) / 2}}{[r]_{p, q} !}\left(\frac{q x u}{3}\right)^{r} \sum_{i=0}^{\infty} \frac{p^{i(i-1) / 2}}{[i]_{p, q} !} \\
& \cdot\left(\frac{x t}{3}\right)^{i} \sum_{k=0}^{\infty} \frac{p^{k(k-1) / 2}}{[k]_{p, q} !}\left(-\frac{x}{3 u t}\right)^{k}
\end{aligned}
$$

$$
=\sum_{i, r, k=0}^{\infty} \frac{(-1)^{k} q\left(\begin{array}{l}
r \\
2
\end{array}\right)\left(\begin{array}{l}
i \\
2
\end{array}\right)+\left(\begin{array}{l}
k \\
2
\end{array}\right)}{[k]_{p, q} ![i]_{p, q} ![r]_{p, q} !}\left(\frac{x}{3}\right)^{k+i+r} u^{r-k} t^{i-k} .
$$

Substituting $r$ by $m+k$ and $i$ by $n+k$ in the above equation, we get the following equality

$$
\begin{aligned}
& \sum_{m, n=-\infty}^{\infty} \sum_{k=0}^{\infty} \frac{(-1)^{k} q}{[k]_{p, q} ![m+k]_{p, q} ![n+k]_{p, q} !}\left(\frac{c}{m+k+1}\right)^{\prime}\left(\begin{array}{c}
n+k \\
2
\end{array}\right)+\left(\begin{array}{l}
k \\
2
\end{array}\right)^{m+n+3 k} u^{m} t^{n} \\
& =\sum_{m, n=-\infty}^{\infty} J_{m, n}^{(8)}(x \mid p, q) u^{m} t^{n} .
\end{aligned}
$$

Explicitly, we obtain the explicit expressions of $(p, q)$ Humbert functions $J_{m, n}^{(6)}(x \mid p, q)$ of the eighth kind as the following power series

$$
\begin{aligned}
& J_{m, n}^{(8)}(x \mid p, q)= \sum_{k=0}^{\infty} \frac{(-1)^{k} q\left(\begin{array}{c}
m+k+1 \\
2
\end{array}\right)_{p}\left(\begin{array}{c}
n+k \\
2
\end{array}\right)+\left(\begin{array}{l}
k \\
2
\end{array}\right)}{[k]_{p, q} ![m+k]_{p, q} ![n+k]_{p, q} !}\left(\frac{x}{3}\right)^{m+n+3 k} \cdot \\
& J_{m, n}^{(8)}(x \mid p, q)= \frac{1}{((p, q) ;(p, q))_{n}((p, q) ;(p, q))_{n}} \\
& \times \sum_{k=0}^{\infty} \frac{\left.(-1)^{k} p\left(\begin{array}{c}
n+k \\
2
\end{array}\right)+\left(\begin{array}{l}
k \\
2
\end{array}\right)\left(\begin{array}{c}
m+k+1 \\
q
\end{array}\right) ;(p, q)\right)_{k}\left(\left(p^{m+1}, q^{m+1}\right) ;(p, q)\right)_{k}\left(\left(p^{n+1}, q^{n+1}\right) ;(p, q)\right)_{k}}{\left(\frac{(p-q) x}{3}\right)^{m+n+3 k}} \\
&= \frac{1}{\Gamma_{p, q}(m+1) \Gamma_{p, q}(n+1)}\left(\frac{x}{3(p-q)}\right)^{m+n} \\
& \times \sum_{k=0}^{\infty} \frac{(-1)^{k} p}{((p, q) ;(p, q))_{k}\left(\left(p^{m+1}, q^{m+1}\right) ;(p, q)\right)_{k}\left(\left(p^{n+1}, q^{n+1}\right) ;(p, q)\right)_{k}}\left(\frac{(p-q) x}{3}\right)^{3 k} \\
&= \frac{\left(\left(p^{m+1}, q^{m+1}\right) ;(p, q)\right)_{\infty}\left(\left(p^{n+1}, q^{n+1}\right) ;(p, q)\right)_{\infty}}{((p, q) ;(p, q))_{\infty}((p, q) ;(p, q))_{\infty}}\left(\begin{array}{c}
k \\
2
\end{array}\right)\left(\begin{array}{c}
m+k+1 \\
2
\end{array}\right) \\
& \times \sum_{k=0}^{\infty} \frac{(-1)^{k} p}{((p, q) ;(p, q))_{k}\left(\left(p^{m+1}, q^{m+1}\right) ;(p, q)\right)_{k}\left(\left(p^{n+1}, q^{n+1}\right) ;(p, q)\right)_{k}}\left(\frac{(p-q) x}{3}\right)^{m+n+3 k} \\
& 2
\end{aligned}
$$


Furthermore, we show the relations between generating functions for the $(p, q)$-Humbert functions.

Theorem 14. The connections between generating functions of the $(p, q)$-Humbert functions of all kinds are given by

$$
\begin{aligned}
& F_{4}(x ; u, t \mid p, q)=F_{3}\left(q x ; u, t \mid \frac{1}{p}, \frac{1}{q}\right), \\
& F_{7}(x ; u, t \mid p, q)=F_{6}\left(q x ; u, t \mid \frac{1}{p}, \frac{1}{q}\right)
\end{aligned}
$$

and

$$
F_{8}(x ; u, t \mid p, q)=F_{5}\left(q x ; u, t \mid \frac{1}{p}, \frac{1}{q}\right)
$$

Further examples can be discussed, but are omitted for the sake of conciseness.

Theorem 15. (Multiplication theorem) The links between the generating functions for the $(p, q)$-Humbert functions of all kinds

$$
\begin{aligned}
& F_{1}(x ; u, t \mid p, q)=F_{3}(x ; u, t \mid p, q) e_{p, q}\left(\frac{q x}{3 u t}\right) e_{p, q}\left(-\frac{x}{3 u t}\right), \\
& F_{3}(x ; u, t \mid p, q)=F_{1}(x ; u, t \mid p, q) E_{p, q}\left(-\frac{q x}{3 u t}\right) E_{p, q}\left(\frac{x}{3 u t}\right), \\
& F_{3}(x ; u, t \mid p, q)=F_{6}(x ; u, t \mid p, q) e_{p, q}\left(\frac{x u}{3}\right) e_{p, q}\left(-\frac{q x u}{3}\right), \\
& F_{6}(x ; u, t \mid p, q)=F_{3}(x ; u, t \mid p, q) E_{p, q}\left(-\frac{x u}{3}\right) E_{p, q}\left(\frac{q x u}{3}\right), \\
& F_{5}(x ; u, t \mid p, q)=F_{7}(x ; u, t \mid p, q) E_{p, q}\left(-\frac{q x}{3 u t}\right) E_{p, q}\left(\frac{x}{3 u t}\right) \\
& F_{7}(x ; u, t \mid p, q)=F_{5}(x ; u, t \mid p, q) e_{p, q}\left(\frac{q x}{3 u t}\right) e_{p, q}\left(-\frac{x}{3 u t}\right) \\
& F_{6}(x ; u, t \mid p, q)=F_{8}(x ; u, t \mid p, q) E_{p, q}\left(-\frac{q x}{3 u t}\right) E_{p, q}\left(\frac{x}{3 u t}\right), \\
& F_{8}(x ; u, t \mid p, q)=F_{6}(x ; u, t \mid p, q) e_{p, q}\left(\frac{q x}{3 u t}\right) e_{p, q}\left(-\frac{x}{3 u t}\right), \\
& F_{7}(x ; u, t \mid p, q)=F_{1}(x ; u, t \mid p, q) E_{p, q}\left(\frac{-x t}{3}\right) E_{p, q}\left(\frac{q x t}{3}\right), \\
& F_{1}(x ; u, t \mid p, q)=F_{7}(x ; u, t \mid p, q) e_{p, q}\left(\frac{x t}{3}\right) e_{p, q}\left(-\frac{q x t}{3}\right) \\
& F_{1}(x ; u, t \mid p, q)=F_{8}(x ; u, t \mid p, q) e_{p, q}\left(-\frac{q x u}{3}\right) E_{p, q}\left(\frac{x u}{3}\right),
\end{aligned}
$$

$$
F_{8}(x ; u, t \mid p, q)=F_{1}(x ; u, t \mid p, q) E_{p, q}\left(\frac{q x u}{3}\right) e_{p, q}\left(-\frac{x u}{3}\right) .
$$

\section{The Recurrence Relations}

In this section, we show the significant interesting recurrence relations for the $(p, q)$-Humbert functions of the first kind so far introduced can be established with respect to $x$ on their generating functions in different ways.

Theorem 16. The $(p, q)$-Humbert functions $J_{m, n}^{(1)}(x \mid p, q)$ satisfy the recurrence relations

$$
\begin{aligned}
& J_{m-1, n}^{(1)}(p x \mid p, q)+p^{n-2 m-1 / 3} q^{2 m-n+1 / 3} J_{m, n-1}^{(1)}\left(p^{2 / 3} q^{1 / 3} x \mid p, q\right) \\
& \quad-p^{-m+n+2 / 3} q^{m+n+2 / 3} J_{m+1, n+1}^{(1)}\left(p^{1 / 3} q^{2 / 3} x \mid p, q\right) \\
& =3 D_{p, q}\left\{J_{m, n}^{(1)}(x \mid p, q)\right\},
\end{aligned}
$$

$$
\begin{aligned}
p^{m-2 n-1 / 3} q^{2 n-m+1 / 3} J_{m-1, n}^{(1)}\left(p^{2 / 3} q^{1 / 3} x \mid p, q\right)+J_{m, n-1}^{(1)}(p x \mid p, q) & \\
& -p^{-m+n+2 / 3} q^{m+n+2 / 3} J_{m+1, n+1}^{(1)}\left(p^{1 / 3} q^{2 / 3} x \mid p, q\right) \\
& =3 D_{p, q}\left\{J_{m, n}^{(1)}(x \mid p, q)\right\},
\end{aligned}
$$

$$
\begin{aligned}
& J_{m-1, n}^{(1)}(p x \mid p, q)+p^{2 n-m-2 / 3} q^{m-2 n+2 / 3} J_{m, n-1}^{(1)}\left(p^{1 / 3} q^{2 / 3} x \mid p, q\right) \\
& \quad-p^{n-2 m-1 / 3} q^{2 m-n+1 / 3} J_{m+1, n+1}^{(1)}\left(p^{2 / 3} q^{1 / 3} x \mid p, q\right) \\
& =3 D_{p, q}\left\{J_{m, n}^{(1)}(x \mid p, q)\right\},
\end{aligned}
$$

$$
\begin{aligned}
& p^{2 n-m-2 / 3} q^{n-2 m+1 / 3} J_{m-1, n}^{(1)}\left(p^{1 / 3} q^{2 / 3} x \mid p, q\right)+J_{m, n-1}^{(1)}(p x \mid p, q) \\
& -p^{m-2 n-1 / 3} q^{2 n-m+1 / 3} J_{m+1, n+1}^{(1)}\left(p^{2 / 3} q^{1 / 3} x \mid p, q\right) \\
= & 3 D_{p, q}\left\{J_{m, n}^{(1)}(x \mid p, q)\right\}
\end{aligned}
$$

$$
\begin{aligned}
p^{m+n-1 / 3} q^{1-m-n / 3} J_{m-1, n}^{(1)}\left(p^{2 / 3} q^{1 / 3} x \mid p, q\right) & \\
& +p^{2 n-m-2 / 3} q^{m-2 n+2 / 3} J_{m, n-1}^{(1)} \\
& \cdot\left(p^{1 / 3} q^{2 / 3} x \mid p, q\right)-J_{m+1, n+1}^{(1)}(p x \mid p, q) \\
= & 3 D_{p, q}\left\{J_{m, n}^{(1)}(x \mid p, q)\right\}
\end{aligned}
$$

$$
\begin{aligned}
p^{2 m-n-2 / 3} q^{n-2 m+2 / 3} J_{m-1, n}^{(1)}\left(p^{1 / 3} q^{2 / 3} x \mid p, q\right) & \\
& +p^{m+n-1 / 3} q^{1-m-n / 3} J_{m, n-1}^{(1)} \\
& \cdot\left(p^{2 / 3} q^{1 / 3} x \mid p, q\right)-J_{m+1, n+1}^{(1)}(p x \mid p, q) \\
= & 3 D_{p, q}\left\{J_{m, n}^{(1)}(x \mid p, q)\right\} .
\end{aligned}
$$


Proof. By applying the $(p, q)$-derivative operator on both sides of Eq. (19), using (15) and (18), we get

$$
\begin{aligned}
\frac{1}{3} & {\left[u e_{p, q}\left(\frac{p x u}{3}\right) e_{p, q}\left(\frac{p x t}{3}\right) e_{p, q}\left(-\frac{p x}{3 u t}\right)\right.} \\
& +t e_{p, q}\left(\frac{q x u}{3}\right) e_{p, q}\left(\frac{p x t}{3}\right) e_{p, q}\left(-\frac{p x}{3 u t}\right) \\
& \left.-\frac{1}{u t} e_{p, q}\left(\frac{q x u}{3}\right) e_{p, q}\left(\frac{q x t}{3}\right) e_{p, q}\left(-\frac{p x}{3 u t}\right)\right] \\
= & \sum_{m, n=-\infty}^{\infty} D_{p, q} J_{m, n}^{(1)}(x \mid p, q) u^{m} t^{n} .
\end{aligned}
$$

Taking $x=p^{2 / 3} q^{1 / 3} x, u=p^{-2 / 3} q^{2 / 3} u$ and $t=p^{1 / 3} q^{-1 / 3} t$ in Eq. (19), then we get the result

$$
\begin{aligned}
p^{1 / 3} q^{-1 / 3} t e_{p, q}\left(\frac{q x u}{3}\right) e_{p, q}\left(\frac{p x t}{3}\right) e_{p, q}\left(-\frac{p x}{3 u t}\right) \\
=\sum_{m, n=-\infty}^{\infty} p^{n-2 m / 3} q^{2 m-n / 3} J_{m, n-1}^{(1)}\left(p^{2 / 3} q^{1 / 3} x \mid p, q\right) u^{m} t^{n} .
\end{aligned}
$$

Using the generating function (19), and taking $x=p^{1 / 3}$ $q^{2 / 3} x, u=p^{-1 / 3} q^{1 / 3} u$ and $t=p^{-1 / 3} q^{1 / 3} t$, we have

$$
\begin{aligned}
& \frac{1}{p^{-2 / 3} q^{2 / 3} u t} e_{p, q}\left(\frac{q x u}{3}\right) e_{p, q}\left(\frac{q x t}{3}\right) e_{p, q}\left(-\frac{p x}{3 u t}\right) \\
& =\sum_{m, n=-\infty}^{\infty} p^{-(m+n / 3)} q^{m+n / 3} J_{m+1, n+1}^{(1)}\left(p^{1 / 3} q^{2 / 3} x \mid p, q\right) u^{m} t^{n} .
\end{aligned}
$$

Using Eqs. (77), (78) and (79), we give the following relation

$$
\begin{aligned}
\frac{1}{3} & {\left[\sum_{m, n=-\infty}^{\infty} J_{m, n}^{(1)}(p x \mid p, q) u^{m+1} t^{n}\right.} \\
& +\sum_{m, n=-\infty}^{\infty} p^{2 m-n-1 / 3} q^{2 m-n+1 / 3} J_{m, n-1}^{(1)}\left(p^{2 / 3} q^{1 / 3} x \mid p, q\right) u^{m} t^{n} \\
& \left.-\sum_{m, n=-\infty}^{\infty} p^{2-m-n / 3} q^{m+n+2 / 3} J_{m+1, n+1}^{(1)}\left(p^{1 / 3} q^{2 / 3} x \mid p, q\right) u^{m} t^{n}\right] \\
& =\sum_{m, n=-\infty}^{\infty} D_{p, q} J_{m, n}^{(1)}(x \mid p, q) u^{m} t^{n} .
\end{aligned}
$$

Thus, we obtain the recurrence relation (71). Similarly, the other equations of this theorem can be proved.
Theorem 17. The $(p, q)$-Humbert functions $J_{m, n}^{(1)}(x \mid p, q)$ have the following recurrence relations

$$
\begin{aligned}
& J_{m-1, n}^{(1)}(p x \mid p, q)+p^{n-2 m-1 / 3} q^{2 m-n+1 / 3} J_{m, n-1}^{(1)}\left(p^{2 / 3} q^{1 / 3} x \mid p, q\right) \\
&= p^{m-2 n-1 / 3} q^{2 n-m+1 / 3} J_{m-1, n}^{(1)}\left(p^{2 / 3} q^{1 / 3} x \mid p, q\right) \\
&+J_{m, n-1}^{(1)}(p x \mid p, q) \\
& \quad-p^{n-2 m-1 / 3} q^{2 m-n+1 / 3} J_{m+1, n+1}^{(1)}\left(p^{2 / 3} q^{1 / 3} x \mid p, q\right) \\
&= p^{2 m-n-2 / 3} q^{n-2 m+2 / 3} J_{m-1, n}^{(1)}\left(p^{1 / 3} q^{2 / 3} x \mid p, q\right)+J_{m, n-1}^{(1)}(p x \mid p, q) \\
&-p^{m-2 n-1 / 3} q^{2 n-m+1 / 3} J_{m+1, n+1}^{(1)}\left(p^{2 / 3} q^{1 / 3} x \mid p, q\right)
\end{aligned}
$$

$$
\begin{aligned}
p^{m+n-1 / 3} q^{1-m-n / 3} J_{m-1, n}^{(1)}\left(p^{2 / 3} q^{1 / 3} x \mid p, q\right) & +p^{2 n-m-2 / 3} q^{m-2 n+2 / 3} J_{m, n-1}^{(1)}\left(p^{1 / 3} q^{2 / 3} x \mid p, q\right) \\
= & p^{2 m-n-2 / 3} q^{n-2 m+2 / 3} J_{m-1, n}^{(1)}\left(p^{1 / 3} q^{2 / 3} x \mid p, q\right) \\
& +p^{m+n-1 / 3} q^{1-m-n / 3} J_{m, n-1}^{(1)}\left(p^{2 / 3} q^{1 / 3} x \mid p, q\right), \\
p^{n-2 m-1 / 3} q^{2 m-n+1 / 3} J_{m, n-1}^{(1)}\left(p^{2 / 3} q^{1 / 3} x \mid p, q\right) & \\
& -p^{-(m+n+2 / 3)} q^{m+n+2 / 3} J_{m+1, n+1}^{(1)}\left(p^{1 / 3} q^{2 / 3} x \mid p, q\right) \\
= & p^{2 n-m-2 / 3} q^{m-2 n+2 / 3} J_{m, n-1}^{(1)}\left(p^{1 / 3} q^{2 / 3} x \mid p, q\right) \\
& -p^{n-2 m-1 / 3} q^{2 m-n+1 / 3} J_{m+1, n+1}^{(1)}\left(p^{2 / 3} q^{1 / 3} x \mid p, q\right) \\
p^{m-2 n-1 / 3} q^{2 n-m+1 / 3} J_{m-1, n}^{(1)}\left(p^{2 / 3} q^{1 / 3} x \mid p, q\right) & -p^{-(m+n+2 / 3)} q^{m+n+2 / 3} J_{m+1, n+1}^{(1)}\left(p^{1 / 3} q^{2 / 3} x \mid p, q\right) \\
= & p^{2 m-n-2 / 3} q^{n-2 m+2 / 3} J_{m-1, n}^{(1)}\left(p^{1 / 3} q^{2 / 3} x \mid p, q\right) \\
& -p^{m-2 n-1 / 3} q^{2 n-m+1 / 3} J_{m+1, n+1}^{(1)}\left(p^{2 / 3} q^{1 / 3} x \mid p, q\right) .
\end{aligned}
$$

Proof. By using (71) and (72), we obtain (81). In similar way, the Eqs. (82), (83), (84) and (85) can be proven.

Theorem 18. The $(p, q)$-Humbert functions $J_{m, n}^{(1)}(x \mid p, q)$ satisfy the following recurrence relations

$$
\begin{aligned}
& 3 \frac{[n]_{p, q}}{x} J_{m, n}^{(1)}(x \mid p, q)=p^{m+n-1 / 3} J_{m, n-1}^{(1)}\left(p^{-(1 / 3)} x \mid p, q\right) \\
& \quad+p^{m+n-1 / 3} q^{n} J_{m+1, n+1}^{(1)}\left(p^{-(1 / 3)} x \mid p, q\right), \\
& 3 \frac{[m]_{p, q}}{x} J_{m, n}^{(1)}(x \mid p, q)=p^{m+n-1 / 3} J_{m-1, n}^{(1)}\left(p^{-(1 / 3)} x \mid p, q\right) \\
& \quad+p^{m+n-1 / 3} q^{m} J_{m+1, n+1}^{(1)}\left(p^{-(1 / 3)} x \mid p, q\right),
\end{aligned}
$$




$$
\begin{aligned}
& 3 \frac{[n]_{p, q}}{x} J_{m, n}^{(1)}(x \mid p, q)=q^{m+n-1 / 3} J_{m, n-1}^{(1)}\left(q^{-(1 / 3)} x \mid p, q\right) \\
& +q^{m+n-1 / 3} p^{n} J_{m+1, n+1}^{(1)}\left(q^{-(1 / 3)} x \mid p, q\right) \\
& 3 \frac{[m]_{p, q}}{x} J_{m, n}^{(1)}(x \mid p, q)=q^{m+n-1 / 3} J_{m-1, n}^{(1)}\left(q^{-(1 / 3)} x \mid p, q\right) \\
& +q^{m+n-1 / 3} p^{m} J_{m+1, n+1}^{(1)}\left(q^{-(1 / 3)} x \mid p, q\right) .
\end{aligned}
$$

Proof. Multiplying both sides of Eq. (22) by $[n]_{p, q}$ and noting that

$$
[n]_{p, q}=p^{-k}[n+k]_{p, q}-p^{-k} q^{n}[k]_{p, q}
$$

and, we get

$$
\begin{aligned}
& {[n]_{p, q} J_{m, n}^{(1)}(x \mid p, q)} \\
& =\sum_{k=0}^{\infty} \frac{(-1)^{k}[n]_{p, q}}{[k]_{p, q} ![m+k]_{p, q} ![n+k]_{p, q} !}\left(\frac{x}{3}\right)^{m+n+3 k} \\
& =\sum_{k=0}^{\infty} \frac{(-1)^{k}\left(p^{-k}[n+k]_{p, q}-p^{-k} q^{n}[k]_{p, q}\right)}{[k]_{p, q} ![m+k]_{p, q} ![n+k]_{p, q} !}\left(\frac{x}{3}\right)^{m+n+3 k} \\
& =\sum_{k=0}^{\infty} \frac{(-1)^{k} p^{-k}[n+k]_{p, q}}{[k]_{p, q} ![m+k]_{p, q} ![n+k]_{p, q} !}\left(\frac{x}{3}\right)^{m+n+3 k} \\
& -\sum_{k=0}^{\infty} \frac{(-1)^{k} p^{-k} q^{n}[k]_{p, q}}{[k]_{p, q} ![m+k]_{p, q} ![n+k]_{p, q} !}\left(\frac{x}{3}\right)^{m+n+3 k} \\
& =\sum_{k=0}^{\infty} \frac{(-1)^{k} p^{-k}}{[k]_{p, q} ![m+k]_{p, q} ![n+k-1]_{p, q} !}\left(\frac{x}{3}\right)^{m+n+3 k} \\
& -\sum_{k=0}^{\infty} \frac{(-1)^{k} p^{-k} q^{n}}{[k-1]_{p, q} ![m+k]_{p, q} ![n+k]_{p, q} !}\left(\frac{x}{3}\right)^{m+n+3 k} \\
& =p^{m+n-1 / 3} \frac{x}{3} \sum_{k=0}^{\infty} \frac{(-1)^{k}}{[k]_{p, q} ![m+k]_{p, q} ![n+k-1]_{p, q} !} \\
& \cdot\left(\frac{p^{-1 / 3} x}{3}\right)^{m+n-1+3 k}+p^{m+n-1 / 3} q^{n} \frac{x}{3} \sum_{k=0}^{\infty} \\
& \cdot \frac{(-1)^{k}}{[k]_{p, q} ![m+k+1]_{p, q} ![n+k+1]_{p, q} !}\left(\frac{p^{-1 / 3} x}{3}\right)^{m+n+3 k+2} \text {. }
\end{aligned}
$$

Using (22) and (91), we obtain (86). Similarly, we can prove (87), (88) and (89).
Theorem 19. The $(p, q)$-Humbert functions $J_{m, n}^{(1)}(x \mid p, q)$ have the following recurrence relations

$$
\begin{gathered}
q^{-(m+n-1 / 3)} J_{m, n-1}^{(1)}\left(q^{1 / 3} x \mid p, q\right) \\
=p^{-(m+n-1 / 3)} J_{m, n-1}^{(1)}\left(p^{1 / 3} x \mid p, q\right) \\
+(p-q) \frac{x}{3} J_{m+1, n}^{(1)}(x \mid p, q), \\
q^{-(m+n-1 / 3)} J_{m-1, n}^{(1)}\left(q^{1 / 3} x \mid p, q\right) \\
=p^{-(m+n-1 / 3)} J_{m-1, n}^{(1)}\left(p^{1 / 3} x \mid p, q\right) \\
\quad+(p-q) \frac{x}{3} J_{m, n+1}^{(1)}(x \mid p, q), \\
q^{-(m+n-2 / 3)} J_{m-1, n-1}^{(1)}\left(q^{1 / 3} x \mid p, q\right) \\
=p^{-(m+n-2 / 3)} J_{m-1, n-1}^{(1)}\left(p^{1 / 3} x \mid p, q\right) \\
\quad+(p-q) \frac{x}{3} J_{m, n}^{(1)}(x \mid p, q),
\end{gathered}
$$

$$
\begin{gathered}
p^{-(m+n-1 / 3)} J_{m, n-1}^{(1)}\left(p^{1 / 3} x \mid p, q\right) \\
=q^{-(m+n-1 / 3)} J_{m, n-1}^{(1)}\left(q^{1 / 3} x \mid p, q\right) \\
\quad-(p-q) \frac{x}{3} J_{m+1, n}^{(1)}(x \mid p, q), \\
p^{-(m+n-1 / 3)} J_{m-1, n}^{(1)}\left(p^{1 / 3} x \mid p, q\right) \\
=q^{-(m+n-1 / 3)} J_{m-1, n}^{(1)}\left(q^{1 / 3} x \mid p, q\right) \\
\quad-(p-q) \frac{x}{3} J_{m, n+1}^{(1)}(x \mid p, q) \\
p^{-(m+n-2 / 3)} J_{m-1, n-1}^{(1)}\left(p^{1 / 3} x \mid p, q\right) \\
=q^{-(m+n-2 / 3)} J_{m-1, n-1}^{(1)}\left(q^{1 / 3} x \mid p, q\right) \\
\quad-(p-q) \frac{x}{3} J_{m, n}^{(1)}(x \mid p, q) .
\end{gathered}
$$

Proof. By (22), we consider

$$
\begin{aligned}
q^{-(m+n-1 / 3)} J_{m, n-1}^{(1)}\left(q^{1 / 3} x \mid p, q\right) \\
=q^{-(m+n-1 / 3)} \sum_{k=0}^{\infty} \frac{(-1)^{k}}{[k]_{p, q} ![m+k]_{p, q} ![n+k-1]_{p, q} !} \\
\quad \cdot\left(\frac{x q^{1 / 3}}{3}\right)^{m+n-1+3 k} \\
=\sum_{k=0}^{\infty} \frac{(-1)^{k}}{[k]_{p, q} ![m+k]_{p, q} ![n+k-1]_{p, q} !}\left(\frac{x}{3}\right)^{m+n-1+3 k} q^{k} .
\end{aligned}
$$

Using the following identity

$$
q^{k}=p^{k}-(p-q)[k]_{p, q}
$$


we get

$$
\begin{aligned}
\sum_{k=0}^{\infty} & \frac{(-1)^{k}}{[k]_{p, q} ![m+k]_{p, q} ![n+k-1]_{p, q} !}\left(\frac{x}{3}\right)^{m+n-1+3 k} q^{k} \\
= & \sum_{k=0}^{\infty} \frac{(-1)^{k}}{[k]_{p, q} ![m+k]_{p, q} ![n+k-1]_{p, q} !}\left(\frac{x}{3}\right)^{m+n-1+3 k} \\
& \cdot\left(p^{k}-(p-q)[k]_{p, q}\right) \\
= & \sum_{k=0}^{\infty} \frac{(-1)^{k} p^{k}}{[k]_{p, q} ![m+k]_{p, q} ![n+k-1]_{p, q} !}\left(\frac{x}{3}\right)^{m+n-1+3 k} \\
& -(p-q) \sum_{k=0}^{\infty} \frac{(-1)^{k}[k]_{p, q}}{[k]_{p, q} ![m+k]_{p, q} ![n+k-1]_{p, q} !}\left(\frac{x}{3}\right)^{m+n-1+3 k} \\
= & p^{-(m+n-1 / 3)} J_{m, n-1}^{(1)}\left(p^{1 / 3} x \mid p, q\right)+(p-q) \frac{x}{3} J_{m+1, n}^{(1)}(x \mid p, q) .
\end{aligned}
$$

Thus, the Eq. (92) is proved. In the same way, equations (93), (94), (95), (96) and (97) can be proved.

Similar recurrence relations can be achieved by using the generating function; in fact, by differentiating with respect to $u$ and $v$, separately, we have:

Theorem 20. The $(p, q)$-Humbert functions satisfy the following properties:

$$
\begin{aligned}
& \frac{x}{3}\left[p^{m-2 n-2 / 3} J_{m, n}^{(1)}\left(p^{2 / 3} x \mid p, q\right)\right. \\
& \left.+p^{-(m+n+1 / 3)} q^{2 m-n-1 / 3} J_{m+2, n+1}^{(1)}\left(q^{1 / 3} x \mid p, q\right)\right] \\
& =[m+1]_{p, q} J_{m+1, n}^{(1)}(x \mid p, q) \text {, } \\
& \frac{x}{3}\left[p^{m-2 n-2 / 3} J_{m+2, n+1}^{(1)}\left(p^{2 / 3} x \mid p, q\right)\right. \\
& \left.+p^{2 m-n-1 / 3} q^{-(m+n+1 / 3)} J_{m, n}^{(1)}\left(p^{1 / 3} q^{1 / 3} x \mid p, q\right)\right] \\
& =[m+1]_{p, q} J_{m+1, n}^{(1)}(x \mid p, q) \text {, } \\
& \frac{x}{3}\left[p^{n-2 m-2 / 3} J_{m, n}^{(1)}\left(p^{2 / 3} x \mid p, q\right)\right. \\
& \left.+p^{-(n+m+1 / 3)} q^{2 n-m-1 / 3} J_{m+1, n+2}^{(1)}\left(p^{1 / 3} q^{1 / 3} x \mid p, q\right)\right] \\
& =[n+1]_{p, q} J_{m, n+1}^{(1)}(x \mid p, q) \\
& \frac{x}{3}\left[p^{n-2 m-2 / 3} J_{m+1, n+2}^{(1)}\left(p^{2 / 3} x \mid p, q\right)\right. \\
& \left.+p^{2 n-m-1 / 3} q^{-(m+n+1 / 3)} J_{m, n}^{(1)}\left(p^{1 / 3} q^{1 / 3} x \mid p, q\right)\right] \\
& =[n+1]_{p, q} J_{m, n+1}^{(1)}(x \mid p, q) .
\end{aligned}
$$

Proof. Differentiating with respect to $u$ in (19), using (15) and (17), we get

$$
\begin{aligned}
& \frac{x}{3}\left[e_{p, q}\left(\frac{p x u}{3}\right) e_{p, q}\left(\frac{x t}{3}\right) e_{p, q}\left(-\frac{p x}{3 u t}\right)\right. \\
& \left.+\frac{1}{u^{2} t} e_{p, q}\left(\frac{q x u}{3}\right) e_{p, q}\left(\frac{x t}{3}\right) e_{p, q}\left(-\frac{p x}{3 u t}\right)\right] \\
& =\sum_{m, n=-\infty}^{\infty}[m]_{p, q} J_{m, n}^{(1)}(x \mid p, q) u^{m-1} t^{n} .
\end{aligned}
$$

Taking $x=p^{2 / 3} x, u=p^{1 / 3} u$ and $t=p^{-2 / 3} t$, we have

$$
\begin{aligned}
& \frac{p^{2 / 3} x}{3} e_{p, q}\left(\frac{p x u}{3}\right) e_{p, q}\left(\frac{x t}{3}\right) e_{p, q}\left(-\frac{p x}{3 u t}\right) \\
& =\sum_{m, n=-\infty}^{\infty} p^{m-2 n / 3} J_{m, n}^{(1)}\left(p^{2 / 3} x \mid p, q\right) u^{m} t^{n} .
\end{aligned}
$$

Setting $x=p^{1 / 3} q^{1 / 3} x, u=p^{-1 / 3} q^{2 / 3} u$ and $t=p^{-1 / 3} q^{-1 / 3} t$, we get

$$
\begin{aligned}
& \frac{p^{4 / 3} x}{3 q^{2 / 3} u^{2} t} e_{p, q}\left(\frac{q x u}{3}\right) e_{p, q}\left(\frac{x t}{3}\right) e_{p, q}\left(-\frac{p x}{3 u t}\right) \\
& \quad=\sum_{m, n=-\infty}^{\infty} p^{-(m+n / 3)} q^{2 m-n / 3} J_{m+2, n+1}^{(1)}\left(p^{1 / 3} q^{1 / 3} x \mid p, q\right) u^{m} t^{n} .
\end{aligned}
$$

Using (105) and by means of the results (106) and (107), we arrive at the following equality:

$$
\begin{aligned}
& \frac{x}{3}\left[\sum_{m, n=-\infty}^{\infty} p^{m-2 n-2 / 3} J_{m, n}^{(1)}\left(p^{2 / 3} x \mid p, q\right) u^{m} t^{n}\right. \\
& \left.\quad+\sum_{m, n=-\infty}^{\infty} p^{-(m+n+1 / 3)} q^{2 m-n-1 / 3} J_{m+2, n+1}^{(1)}\left(q^{1 / 3} x \mid p, q\right) u^{m} t^{n}\right] \\
& \quad \cdot \sum_{m, n=-\infty}^{\infty}[m+1]_{p, q} J_{m+1, n}^{(1)}(x \mid p, q) u^{m} t^{n} .
\end{aligned}
$$

Thus, we obtain the result (101). Proceeding on parallel lines as mentioned above, the relations (102), (103) and (104) are immediate consequences of the definitions (19), (15) and (17).

\section{Conclusion and Perspectives}

The $(p, q)$-Humbert functions or the twin-basic Humbert functions have various applications in the field of mathematical physics and engineering sciences and so on. There are some results that have been noticed in this study. We have seen some particular cases of $(p, q)$-Humbert functions of the first kind that can be introduced belonging to the family of $(p, q)$-Humbert functions. The $(p, q)$-Humbert functions of the first kind allow us to describe many aspects of computational analysis. It is also interesting to explore how these classes of $(p, q)$-Humbert functions of the first kind can be described in terms of $(p, q)$-Humbert functions of the 
different types. Many properties of these new transforms have been proved and should be a starting point of many other works. For this, the researchers recommended to study these other seven families of $(p, q)$-Humbert functions from these extensions as a parallel study of this work. Further work will be carried out in the next future in other fields of interest.

\section{Data Availability}

No data were used to support this paper.

\section{Conflicts of Interest}

The author of this paper declare that they have no conflicts of interest.

\section{Funding}

The author received no specific funding for this work.

\section{Acknowledgments}

The author thanks the anonymous referees for the careful revision of the manuscript. Their comments and suggestions have substantially improved the quality of the paper.

\section{References}

[1] M. Acikgoz, S. Araci, and U. Duran, "Some $(p, q)$-analogues of Apostol type numbers and polynomials," Acta et Commentationes Universitatis Tartuensis de Mathematica, vol. 23, no. 1, pp. 37-50, 2019.

[2] S. Araci, U. Duran, M. Acikgoz, and H. M. Srivastava, "A certain $(p, q)$-derivative operator and associated divided differences," Journal of Inequalities and Applications, vol. 2016, no. $1,2016$.

[3] I. M. Burban and A. U. Klimyk, "P,Q-differentiation, $(P, Q)$ -integration, and $(P, Q)$-hypergeometric functions related to quantum groups," Integral Transforms and Special Functions, vol. 2, no. 1, pp. 15-36, 1994.

[4] V. Kac and P. Cheung, Quantum calculus, Springer, 2001.

[5] C. Kızılateş, N. Tuğlu, and B. Çekim, "On the $(p, q)$-Chebyshev Polynomials and Related Polynomials," Mathematics, vol. 7, no. 2, p. 136, 2019.

[6] V. Singh, M. A. Khan, and A. H. Khan, "The characterization properties and basic hypergeometric functions of $(p, q)$-analogue," Palestine Journal of Mathematics, vol. 9, pp. 220-230, 2020.

[7] U. Duran, M. Acikgoz, and S. Araci, "Unified ( $p, q)$-analog of Apostol type polynomials of order $\alpha$," Univerzitet $u$ Nišu, vol. 32, no. 1, pp. 1-9, 2019.

[8] U. Duran, M. Acikgoz, A. Esi, and S. Araci, "A note on the $(p, q)$-Hermite polynomials," Applied Mathematics \& Information Sciences, vol. 12, no. 1, pp. 227-231, 2018.

[9] U. Duran, M. Acikgoz, and S. Araci, "On $(p, q)$-Bernoulli, $(p, q)$-Euler and $(p, q)$-Genocchi polynomials," Journal of Computational and Theoretical Nanoscience, vol. 13, no. 11, pp. 7833-7846, 2016.

[10] U. Duran, M. Acikgoz, and S. Araci, "On some polynomials derived from $(p, q)$-Calculus," Journal of Computational and Theoretical Nanoscience, vol. 13, no. 11, pp. 7903-7908, 2016.
[11] J. Jagannathan, “ $(P, Q)$-Special functions, Special Functions and Differential Equations," in Proceedings of a Workshop held at The Institute of Mathematical Sciences, pp. 13-24, Matras, India, January, 1997.

[12] R. Jagannathan and K. Srinivasa Rao, "Two-parameter quantum algebras, twin- basic numbers, and associated generalized hypergeometric series,” 2006, https://arxiv.org/abs/0602613.

[13] V. Sahai and S. Yadav, "Representations of two parameter quantum algebras and $p, q$-special functions," Journal of Mathematical Analysis and Applications, vol. 335, no. 1, pp. 268279, 2007.

[14] P. N. Sadjang, "On two $(p, q)$-analogues of the Laplace transform," Journal of Difference Equations and Applications, vol. 23, no. 9, pp. 1-23, 2017.

[15] P. N. Sadjang, "On the Fundamental Theorem of $(p, q)$-Calculus and Some $(p, q)$-Taylor Formulas," Results in Mathematics, vol. 73, no. 1, p. 39, 2018.

[16] P. N. Sadjang, "On $(p, q)$-Appell polynomials," Analysis Mathematica, vol. 45, no. 3, pp. 583-598, 2019.

[17] M. Mursaleen, K. J. Ansari, and A. Khan, "On $(p, q)$-analogue of Bernstein operators," Applied Mathematics and Computation, vol. 266, pp. 874-882, 2015, Erratum: 276 (2016), pp. 70-71.

[18] M. Mursaleen, F. Khan, and A. Khan, "Approximation by $(p, q)$-Lorentz polynomials on a compact disk," Complex Analysis and Operator Theory, vol. 10, no. 8, pp. 1725-1740, 2016.

[19] K. Khan and D. K. Lobiyal, "Bèzier curves based on Lupaş $(p, q)$-analogue of Bernstein functions in CAGD," Journal of Computational and Applied Mathematics, vol. 317, pp. 458477, 2017.

[20] B. R. Pasricha, "Some integrals involving Humbert function," Proceedings of the Indian Academy of Sciences - Section A, vol. 18, no. 1, pp. 11-19, 1943.

[21] R. S. Varma, "On Humbert functions," The Annals of Mathematics, vol. 42, no. 2, pp. 429-436, 1941.

[22] H. M. Srivastava and A. Shehata, "A family of new q-Extensions of the Humbert functions," European Journal of Mathematical Sciences, vol. 4, no. 1, pp. 13-26, 2018.

[23] H. M. Srivastava, G. Yasmin, A. Muhyi, and S. Araci, "Certain results for the twice-iterated $2 \mathrm{D} q$-Appell polynomials," Symmetry, vol. 11, no. 10, p. 1307, 2019.

[24] G. Brodimas, A. Jannussis, and R. Mignani, Two-parameter quantum groups, vol. 820, Universita di Roma Preprint, 1991.

[25] R. Chakrabarti and R. Jagannathan, “A $(p, q)$-oscillator realization of two-parameter quantum algebras," Journal of Physics A Mathematical and General, vol. 24, no. 13, pp. L711-L718, 1991. 